\title{
Nanocrystal integrated light emitting diodes based on radiative and nonradiative energy transfer for the green gap
}

\author{
Sedat Nizamoglu ${ }^{1,3,4}$, Emre Sari ${ }^{1,3,4}$, Jong-Hyeob Baek ${ }^{5}$, In-Hwan Lee ${ }^{6}$ and Hilmi Volkan Demir ${ }^{1,2,3,4, *}$ \\ ${ }^{1}$ Department of Electrical and Electronics Engineering, ${ }^{2}$ Department of Physics, ${ }^{3}$ Nanotechnology Research Center, ${ }^{4}$ Institute of Materials \\ Science and Nanotechnology, Bilkent University, Bilkent, Ankara 06800 Turkey \\ ${ }^{5}$ Korea Photonics Technology Institute, Gwangju 500-460, Korea \\ ${ }^{6}$ School of Advanced Materials Engineering, Chonbuk National University, Chonju 561-756, Korea \\ *Tel:[+90](312)2901021,e-mail: volkan@bilkent.edu.tr
}

Recently the photometric conditions for ultra-efficient solid-state lighting have been discussed [1-2]. These studies show that a luminous efficacy of optical radiation at $408 \mathrm{~lm} / \mathrm{W}_{\text {opt }}$ and a color rendering index (CRI) of 90 at a correlated color temperature (CCT) of $3000 \mathrm{~K}$ are achievable at the same time. For this purpose light emitting diodes (LEDs) emitting in blue, green, yellow, and red colors at 463, 530, 573, and $614 \mathrm{~nm}$ with relative optical power levels of $1 / 8,2 / 8,2 / 8$, and 3/8, are required, respectively [1-2]. Although $\operatorname{In}_{\mathrm{x}} \mathrm{Ga}_{1-\mathrm{x}} \mathrm{N}$ material system is capable to cover the whole visible by changing the In composition $(x)$, it is technically extremely challenging to obtain efficient green/yellow light emitting diodes especially at those wavelengths (i.e., at $530 \mathrm{~nm}$ and $573 \mathrm{~nm}$, respectively) due to reduced internal quantum efficiency [2-4]. Furthermore, by using the $\left(\mathrm{Al}_{\mathrm{x}} \mathrm{Ga}_{1-\mathrm{x}}\right)_{1-\mathrm{y}} \mathrm{In}_{\mathrm{y}} \mathrm{P}$ quaternary alloy it is also possible to cover from $650 \mathrm{~nm}$ to $580 \mathrm{~nm}$. However, the efficiencies significantly decrease towards green. Therefore, there exists a significant gap in the green-yellow spectral regions (known as "the green gap") to make efficient light emitting diodes. To address this green gap problem, we propose and demonstrate proof-of-concept nanocrystal (NCs) hybridized green/yellow light emitting diodes that rely on both radiative energy transfer and nonradiative energy transfer (i.e., FRET- Förster resonance energy transfer) for color conversion on near-ultraviolet (near-UV) LEDs.

In this paper we present the design, growth, fabrication, integration and characterization of these nanocrystal integrated light emitting diodes. In these NC-LED we use direct and FRET-enhanced color conversion, and also investigate FRET-pumped light emitting structures. In our first direct color conversion NC-LED, we use greenemitting CdSe $/ \mathrm{ZnS}$ core/shell nanocrystals $\left(\lambda_{\mathrm{PL}}=548 \mathrm{~nm}\right)$ on near-UV InGaN $/ \mathrm{GaN}$ LEDs $\left(\lambda_{\mathrm{EL}}=379 \mathrm{~nm}\right)$. In our second FRET-enhanced color conversion NC-LED, we achieve a quantum efficiency enhancement of $9 \%$ for the integrated NC layer with respect to NC layer of the first NC-LED. For that we use a custom-design assembly of cyan- and green-emitting CdSe/ZnS core/shell nanocrystals $\left(\lambda_{\mathrm{PL}}=490 \mathrm{~nm}\right.$ and $548 \mathrm{~nm}$, respectively) to recycle the trapped excitons into nanocrystals via FRET. Finally, we examine FRET directly from epitaxial quantum wells to colloidal nanocrystals in their close proximity. Using FRET-pumping in color conversion, a potential efficiency enhancement is predicted in such hybrid LEDs.

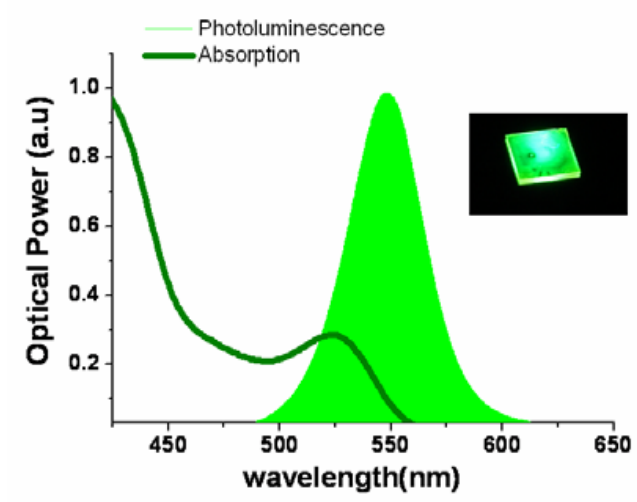

(a)

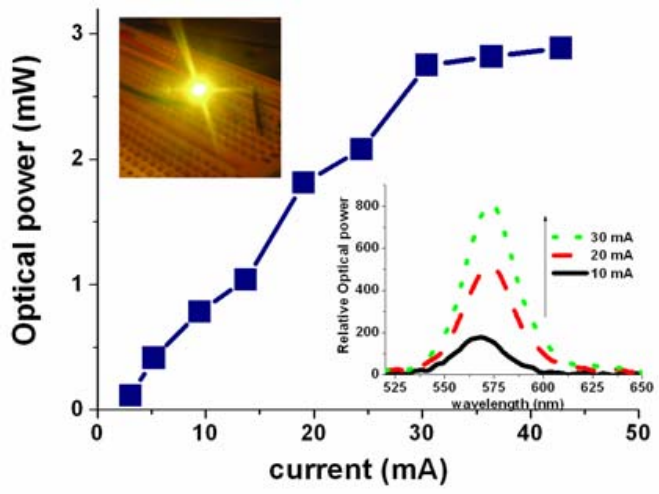

(b)

Figure 1. (a) The emission and absorption spectra of green-emitting $\mathrm{CdSe} / \mathrm{ZnS}$ core/shell nanocrystals along with their photoluminescence picture given in the inset and (b) the total optical power of green nanocrystals $\left(\lambda_{\mathrm{PL}}=548 \mathrm{~nm}\right)$ hybridized on near-UV LED $\left(\lambda_{\mathrm{EL}}=379 \mathrm{~nm}\right)$ at different levels of current injection (with no heat sink or active cooling), along with the emission spectra and picture of the hybrid NC-LED when electrically driven at room temperature.

For the first NC-LED, we integrate $9.1 \mathrm{nmol}$ green-emitting CdSe/ZnS core/shell nanocrystals shown in Figure 1 (a) as the color converters on our near-UV LED to fully down-convert the LED electroluminescence at $379 \mathrm{~nm}$. In Figure 1(b), as the current injection level increases, the optical output power of our LED raises as expected. When the current level reaches $19.05 \mathrm{~mA}$, the optical power becomes $1.81 \mathrm{~mW}$, which corresponds to a quantum efficiency of $46 \%$ for the integrated nanocrystal layer. The resultant luminescence of the hybrid LED is shown as 
a function of increasing current injection level in the inset of Figure 1(b). Here it is important to note that the green NC emission shifted $20 \mathrm{~nm}$ from $548 \mathrm{~nm}$ to $568 \mathrm{~nm}$ in the solid film. This is mainly due to the nonradiative energy transfer among nanocrystals because of their size distribution $(<5 \%)$. This red shift can be easily compensated for using NCs with a shorter starting peak emission wavelength.

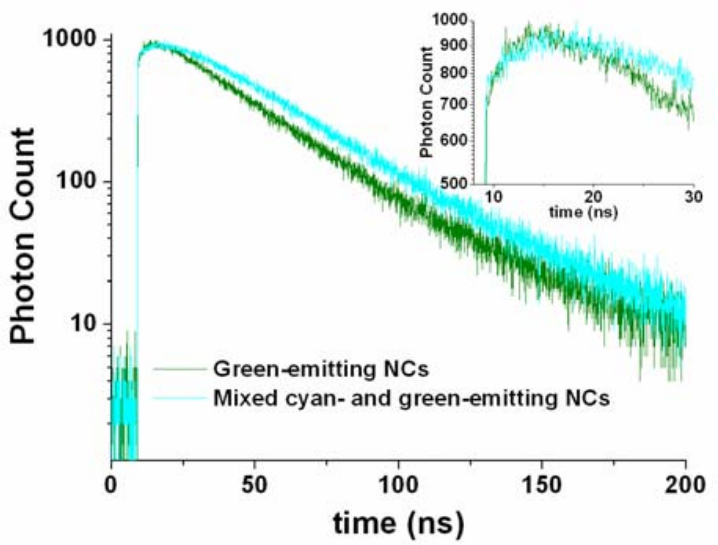

(a)

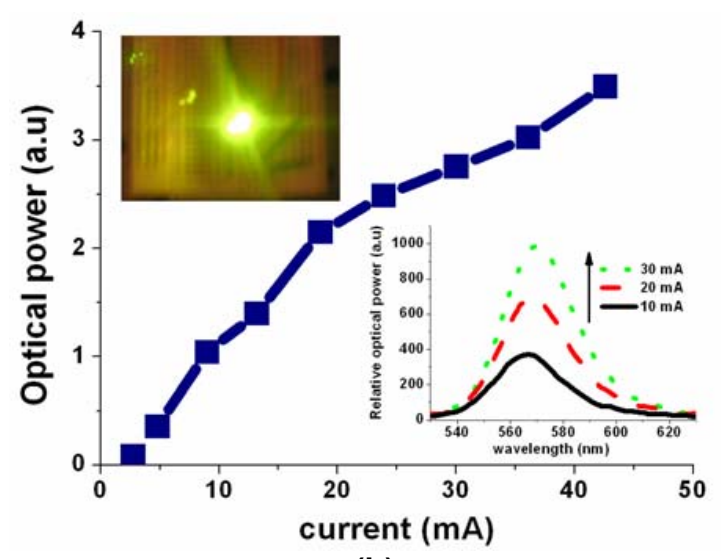

(b)

Figure 2. (a) Time-resolved spectroscopy measurements of the integrated green-emitting CdSe/ZnS core/shell nanocrystals $\left(\lambda_{P L}=548 \mathrm{~nm}\right)$ and mixed cyan- and green-emitting CdSe/ZnS core/shell nanocrystals $\left(\lambda_{P L}=490\right.$ and $548 \mathrm{~nm}$, respectively) on near-UV LED $\left(\lambda_{E L}=379 \mathrm{~nm}\right)$ and (b) the total optical power of mixed cyan- and green-emitting NCs $\left(\lambda_{P L}=490 \mathrm{~nm}\right.$ and $548 \mathrm{~nm}$, respectively) hybridized on near-UV LED $\left(\lambda_{E L}=379 \mathrm{~nm}\right)$ at different levels of current injection (with no heat sink or active cooling), along with the emission spectra and picture of the resulting FRET-NC-LED when electrically driven at room temperature.

For our second FRET-enhanced color conversion NC-LED, we integrate 3.4 nmol cyan-emitting nanocrystals and 4.9 nmol green-emitting nanocrystals as an energy gradient structure on a similar near-UV LED. We use such an integrated NC layer to increase the efficiency of the hybrid NC layer by recycling the trapped excitons in defect centers. In Figure 2(a) the time-resolved spectroscopy measurements of mixed cyan- and green-emitting nanocrystal film is shown and the energy rise (i.e., the exponential increase) in the energy gradient structure of mixed cyan-green nanocrystals is more dominant with respect to the case of only green nanocrystals. This is because of the strong exciton migration to green-emitting nanocrystals. Consequently the quantum efficiency of the nanocrystal film is enhanced by $9 \%$. In Figure 2(b) the resulting luminescence of the hybrid devices is shown. As a result of the enhanced quantum efficiency of the mixed nanocrystal assembly, at $19.05 \mathrm{~mA}$ the FRET-enhanced NC-LED reaches a luminous efficiency of $19 \mathrm{~lm} / \mathrm{W}$, while the luminous efficacy of optical radiation is maintained at $425 \mathrm{~lm} / \mathrm{W}_{\text {opt }}$.

In our final demonstration, we show nonradiative pumping of the nanocrystals by using Förster resonance energy transfer. Energy transfer pumping from quantum wells to nanocrystals allows for direct excitation of the nanocrystals that lead to a subsequent single-step radiative recombination process. If we compare this with radiative pumping through electroluminescence, the radiative pumping requires two subsequent steps of radiative recombination. The use of nonradiative energy transfer allows us to make color-converted FRET-enhanced LEDs with an external quantum efficiency of $22 \%$, starting with a state-of-the-art near-UV LED that has an external quantum efficiency of 40\%. Furthermore, using FRET-pumping as an additional pathway to transfer excitation from the LED to the nanocrystals, even higher external quantum efficiencies are expected.

In conclusion, we presented proof-of-concept demonstrations of green/yellow solid state lighting that utilizes FRET-enhanced color conversion and FRET-pumping. These results reveal that nanocrystal based solid state lighting holds great promise for future efficient lighting applications.

Acknowledgements: This work is supported by TUBITAK 104E114, 106E020, 107E088, 107E297, and 109E002, EU MOON, EU NoE PHOREMOST. HVD acknowledges additional support from European Science Foundation European Young Investigator Award (ESFEURYI) and Turkish Academy of Sciences Distinguished Young Scientist Award (TUBA GEBIP).

References

[1] J. M. Phillips et al., Laser \& Photon. Rev., 1, 307-333, 2007.

[2] J. Y. Tsao, The 21st Annual Meeting of The IEEE Lasers \& Electro-Optics Society Conference Proceedings, WU4, 2008.

[3] M. R. Krames et al., J. Disp. Technol., 3, 160-175, 2007.

[4] S. Nakamura and G. Fasol, The Blue Laser Diode. Berlin: Springer, 1997.

[5] S. Nizamoglu et al., IEEE Journal of Special Topics in Quantum Electronics (in press). 